\title{
On Processing Methods and Skills of Trapezoidal Thread by Means of CNC Lathe
}

\author{
Zengmei Song* and Weizhang Wang \\ College of Mechanical and Electrical Engineering, Weifang University, Weifang China \\ ${ }^{*}$ Corresponding author
}

\begin{abstract}
Big size of both pitch and thread form of trapezoidal thread and high accuracy tend to cause serious tool tip wear, and even breaking edge and tipping due to deep penetration, fast feeding and large hogging. High-efficient trapezoidal thread turning by the CNC remains a mechanical problem for the time being. This thesis analyzes trapezoidal thread CNC processing in detail and concludes that stratified cutting by means of left and right cutting method is advisable. Turning tool material and parameters are considered with conclusion that application of hard alloy turning tools is suitable for trapezoidal thread CNC processing. Factors to be considered in grinding trapezoidal thread CNC turning tool are also given. Finally ingenious application of sub-program leads to trapezoidal thread stratified processing on the basis of processing technology analysis of trapezoidal thread CNC lathe.
\end{abstract}

Keywords-CNC lathe; trapezoidal thread; stratified cutting;sub-program

\section{INTRODUCTION}

The thread is shaped along the helix on the surface of the cylindrical workpieces.

With continuous salient and groove of the same profile. Workpieces with thread are widely used in mechanical manufacturing. Turning in thread processing is rather common at present, and the thread is usually processed by the CNC lathe in modernized factories. Automatic processing of the thread is the basic function of the CNC lathe. It differs much from ordinary lathes in that feeding of the tool holder driven by the servomotor is controlled by the CNC system, combining with rotation of the spindle and processes spread as required. The thread can be automatically processed by the CNC system if the starting point coordinate, the terminal point coordinate and the thread pitch are given in programming. ${ }^{[1][2]}$ The thread is actually formed by the simultaneous motion of linear motion of the cutting tool and that of the spindle, rotating in proportion input beforehand. For instance, ordinary triangle thread can multiply efficiency compared with horizontal lathe. But high-speed turning of big pitch trapezoidal thread by CNC lathe faces a lot of difficulties. Processing difficulties of trapezoidal thread consist of big size of both pitch and thread form, high accuracy, small roughness of both tooth type surfaces, which lead to deep penetration, fast feeding and large hogging, simultaneous tool feeding of the three cutting edges, and thus causes vibration.

During processing, the big length of cutting edge involved and the cutting heat lead to big cutting force. Unreasonable technology arrangement may cause serious tool tip wear, and even breaking edge and tipping, thus seriously affecting processing accuracy because of the cutting heat and excessive cutting force. How to process trapezoidal thread with both high speed and high efficiency by means of CNC lathe remains a problem in mechanical processing. ${ }^{[3]}$

\section{Processing Technology Analysis of TRAPEZOIDAL THREAD CNC LATHE}

Advantages of the CNC lathe, high accuracy and high efficiency, cannot be practically realized without reasonable processing technology, so detailed analysis of the trapezoidal thread processing technology is necessary.

\section{A. Selection of Trapezoidal Thread Processing Method}

In thread processing of the CNC lathe popular methods contain direct way, oblique way, left and right cutting method and rough grooving method. Below is analysis of thread processing methods considered above so as to find a way most suitable for trapezoidal thread.

Direct way: The whole length of the threading tool feeds intermittently. In this way all the three sides of the threading tool are involved in cutting, which makes chip-removal difficult and increases both cutting force and cutting heat, and thus serious tool tip wear. Breaking edge and tipping may occur when the cutting tool is fed too much. G92 instruction of CNC lathe can be achieved this way, but obviously this is not advisable.

Oblique way: The whole length of the threading tool feeds intermittently along the thread angle oblique direction. Because of one side edge working, this trapezoidal thread processing method may cause cutting edge wear and the surface of the thread processed is less straight, so the accuracy of the thread form is less ideal. At the same time, when working with one side edge, cutting depth is calculated by the control system, low cutting tool load can make chip-removal easier. Because of good processing condition of the side edge and easy chip removal, this processing method is convenient for trapezoidal thread processing when accuracy required is not so high. Instruction G76 of the CNC lathe can be achieved this way.

Left and right cutting method: The threading tool feeds into the depth of the alternative gap along the thread angle. This method is similar to oblique way stated above and is generally achieved by the CNC lathe by means of macro instruction. Left 
and right cutting method can achieve cutting of three directions, left, middle and right.

It achieve cutting with one side edge or two and can avoid disadvantages of cutting with three side edges simultaneously, and processing effect is especially ideal when processing long-pitch trapezoidal thread this way. Besides left-right cutting tool driving, stratified cutting is also needed because of big threading tool depth.

Rough grooving method: First process thread groove roughly with grooving tool, and then process the two sides of the thread with trapezoidal cutting tool. Programming and processing of this method is rather difficult to achieve by the CNC lathe.

According to the analysis above, stratified cutting of left and right cutting method is relatively advisable when processing trapezoidal thread with the CNC lathe.

Thread cutting instruction G32, G92, G76 are among the thread loop instructions from system FANAUC. Judged according to the cutting path of the instruction, no single instruction of any kind can achieve left-right stratified cutting. Program adjustment is needed to meet processing technology requirements of trapezoidal thread by using existing instructions. ${ }^{[4]}$

\section{B. Cutting Tool Materials and Parameters Selection of Trapezoidal Thread CNC Processing}

1) Selection of cutting tool materials

High-speed steel, cemented carbide, coated carbide and so on are all among the cutting material types. Type of material to process is critical for selection of proper cutting tool, and material grade is determined by different processing stages. Cemented carbide is advisable for steel thread, for this cutting tool material is suitable for both rough processing and finishing processing and is suitable for trapezoidal thread processing by means of the CNC lathe.

\section{2) Geometric angle of the cutting tool}

Processing error of the thread is related to the angle of grind thread cutting tool.

The front angle influences the thread form angle of the trapezoidal thread, as thread form angle cut by the thread cutting tool with front angle is greater than angle of tool nose of the turning tool, and the greater the front angle, the greater the thread form angle. Therefore modification of the tool nose of the turning tool is necessary to assure accuracy of the thread form angle.

According to experience, if the front angle of the turning tool is less than $12^{\circ}$, the value of the tool nose angle can be calculated according to the approximate formulas below ${ }^{[5]}$

$$
\beta=\alpha \cdot \cos \gamma
$$

In which $\alpha$ stands for the thread form angle of the trapezoidal thread, and $\alpha=30^{\circ} ; \beta$ refers to the tool nose angle, and $\gamma$ the front angle.
Clearance angle of the turning tool also exerts much influence on the processing accuracy of the trapezoidal thread. When turning the movement of the turning tool is spiral movement relative to that of the workpieces. Spiral movement may affect the practical clearance angle of the side edges when the turning tool is at work, which can be neglected when turning the excircle because of the relative small feed, but outstanding when turning trapezoidal thread, which is determined by the spiral angle.

Calculation formula of the spiral angle is as below:

$$
\tan \omega=\frac{L}{\pi \cdot D}
$$

Operation clearance angle of the side edge in maleic feeding direction is:

$$
\alpha_{h}=\left(3^{\circ} \sim 5^{\circ}\right)+\omega
$$

And the operation clearance angle of the side edge in reverse feeding direction:

$$
\alpha_{h}=\left(3^{\circ} \sim 5^{\circ}\right)-\omega
$$

In which $\omega$ is the spiral angle; $\alpha_{h}$ clearance angle of the turning tool; $L$ pitch of the trapezoidal thread, mm; $D$ big diameter of the trapezoidal, $\mathrm{mm}$.

Therefore when grinding the CNC turning tool of processing the trapezoidal thread, changes of the operation clearance angle must be taken into consideration.

\section{Installing the Trapezoidal Thread Cutting Tool}

In trapezoidal thread processing, tool setting is necessary because of differences of the length of the cutting tool installed to assure the same cutter location and workpiece coordinate origin in the machine coordinate system. The coordinate of the cutter location of the trapezoidal in the machine coordinate system is given below:

value of the machine coordinate $=$ reference bias + cutting tool bias+coordinate value of workpieces

Application of formula(5)above can achieve tool setting by cutting tool bias adjustment to assure cutter location coincidence of each cutting tool at certain ideal location, and this is the nature of tool setting of the CNC lathe. Application of formula(5)can assure each trapezoidal thread cutting tool to accurately process thread at the same tool start point each time without thread disorder on condition of the same cutter location of each cutting tool after tool changing.

\section{ANALysis Of TRAPEZOIDAl ThreAd PROCESSING PROGRAMMING}

Parameters of machined parts by using the CNC lathe to process the trapezoidal thread is shown as Figure 1. Call 
sub-program for processing programming to achieve left-right cutting.

The trapezoidal thread below can be processed by thread cutting loop instructions G76, but this faces certain technical difficulty. According to long-term experience, processing the trapezoidal thread by calling sub-program for stratified cutting with thread cutting instruction G32 may achieve good effect with safety and reliability.

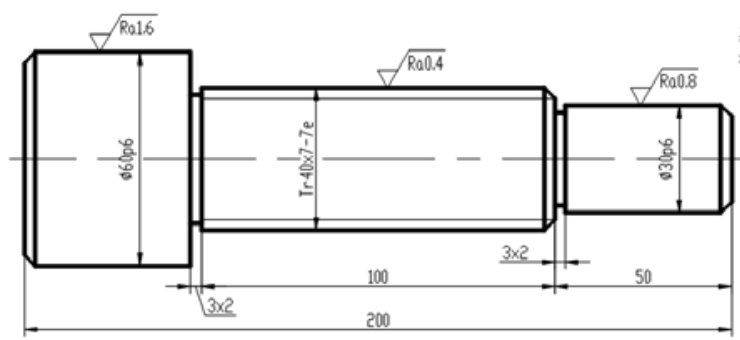

FIGURE I. SCHEMATIC DIAGRAM OF PROCESSING WORKPIECE

Calculation results: trapezoidal thread minor diameter: $\mathrm{d}_{1}=32 \mathrm{~mm}$; mid-diameter: $\mathrm{d}_{2}=36.5 \mathrm{~mm}$; thread bottom width: $\mathrm{w}=2.562 \mathrm{~mm}$; thread tooth type depth: $\mathrm{H}=4 \mathrm{~mm}$.

Tool head width being given $\mathrm{D}=2 \mathrm{~mm}$ on the basis of the tooth bottom width, the offset can be derived, $0.562 \mathrm{~mm}$. Mid diameter tolerance is offered the intermediate value according to the calculation result. The given offset is $0.6 \mathrm{~mm}$ considering the tool head wearing. The offset can be slightly reduced if finishing processing allowance is put into consideration. Adjust the program after processing and calculate the accurate offset based on measurement of the intermediate diameter value, and then accomplish the final processing.

Select cutting depth of each step according to the total cutting depth. Call sub-program for trapezoidal thread rough processing, rough cutting frequency, 77; call sub-program for trapezoidal thread finishing processing, finishing cutting frequency, 77.

Processing program of the trapezoidal thread is as below:

Main program

00003

M03 S300;

T0101;

G00 X48 Z5;(48 is the value of roughcast location point X)

M08;

M98 P770004;(77 is the rough cutting frequency)

M09;

T0100

G28;

T0202;

G00 X48 Z5;
M08

M98 P770004;(is the finishing cutting frequency)

M09;

T0200;

M30;

(2)Subprogram

$\mathrm{O} 0004$

G00 U-8.4;(8.4 is the value of $\mathrm{U}$ )

G32 U0 Z-108 F8;

G00 U8.4;

Z4.73;

U-8.4;

G32 U0 Z-108 F8;

G00 U8.4;

Z5.26;

U-8.4;

G32 U0 Z-108 F8;

G00 U8.3;

Z5;

M99;

\section{CONCLUSIONS}

The following conclusions are drawn from the analysis above.

1) Stratified cutting of left and right cutting method is relatively advisable when processing trapezoidal thread with the CNC lathe. Program adjustment is needed to meet processing technology requirements of trapezoidal thread by using existing instructions.

2) Application of hard alloy turning tools is suitable in trapezoidal thread CNC processing.

3) Processing error of the thread is related to the angle of grind thread cutting tool.

4) Qualified trapezoidal thread can be processed safely and reliably by calling for sub-program for stratified processing.

\section{REFERENCES}

[1] Yu Yingliang, "NC processing programming \&operation[M]”, Higher education publishing house, 2005.

[2] Gouqi, "MadterCAM five axis processing method”, Mechanical industry press, 2004.

[3] Peng Deyin and Aijun, “Turnery technology and technique training”, China labor and social insurance press, 2001.

[4] Zhang Chaoying and Luo Xueke, "CNC lathe processing technology, programming and operation”, Higher education publishing house, 2003.

[5] Feng Xiaoping, “CNC lathe programming and operation”, Mechanical industry press, 2004. 